\title{
Mapping complex polarization states of light on a solid
}

\author{
M. Alameer, A. Jain, M. G. Rahimian, H. Larocque, P. B. Corkum, E. Karimi, (i) and V. R. Bhardwaj* \\ Department of Physics, University of Ottawa, Ontario K1N 6N5, Canada \\ *Corresponding author: ravi.bhardwaj@uottawa.ca
}

Received 28 September 2018; accepted 17 October 2018; posted 26 October 2018 (Doc. ID 346986); published 21 November 2018

\begin{abstract}
Polarization states of light, represented by different points on a Poincaré sphere, can be readily analyzed for a Gaussian beam by a combination of wave plates and polarizers. However, this method cannot be extended to higher-order Poincaré spheres and complex polarization patterns produced by coherent superpositions of vector vortex (VV) beams. We demonstrate the visualization of complex polarization patterns by imprinting them onto a solid surface in the form of periodic nano-gratings oriented parallel to the local structure of the electric field of light. We design unconventional surface structures by controlling the superposition of $\mathrm{VV}$ beams. Our method is of potential interest to the production of sub-wavelength nano-structures. (๑) 2018 Optical Society of America
\end{abstract}

https://doi.org/10.1364/OL.43.005757

Light-matter interactions are governed by the polarization state of light. In solids, the spatial periodic surface structures induced by the Gaussian laser beams are strongly correlated with the laser polarization $[1,2]$. In the domain of attosecond technology, laser polarization influences the generation of higher-order harmonics of the fundamental laser beam, both in solid and gas phases, by manipulating the trajectories of the ionized electrons $[3,4]$. In circular dichroism $[5,6]$, the differential absorption of light by chiral molecules (liquid or gas phase) also depends on the polarization state of light. Light-matter interactions involving non-Gaussian intensity profiles such as optical vector vortex (VV) [7] beams with complex states of polarization (SoPs) have recently been investigated due to their potential applications. For example, first-order VV beams such as tightly focused radially polarized beams were used in particle acceleration [8], spectroscopy [9], optical trapping, and manipulation [10]. In general, a thorough knowledge of SoPs, therefore, will enable us to properly understand the light-matter interaction process.

Pure SoPs of light can be represented by points on the surface of a Poincaré sphere [11]. For a Gaussian beam, all the points on the equator of the sphere represent linear polarizations, while the poles represent the handedness of circular polarization. Other points on the sphere represent elliptically polarized light. SoPs of light represented by such a zeroorder Poincaré sphere can be readily analyzed by a combination of wave plates and polarizers. Extending this technique to characterize higher-order Poincaré spheres requires measuring the four Stokes parameters related to the intensities of six different zero-order polarization states using a spatially resolved detector. Using polarization tomography, the output polarization state can be reconstructed from the measured spatially dependent intensity profiles [12]. However, direct visualization of the higher-order Poincaré spheres is not feasible.

To visualize a polarization pattern, light has to interact with a medium that allows the pattern to be spatially resolved. In recent years, laser structuring of materials has been used to characterize the SoPs of Gaussian (linear) and first-order vortex beams (radial and azimuthal) $[13,14]$. Periodic grating-like structures were induced by the laser within the ablation craters, as well as in the bulk of solids [1,2]. Primarily, patterns were observed to be oriented parallel [15] (perpendicular [16,17]) to the laser polarization on the surface (inside the bulk) of the material, although both orientations have also been reported on the surface $[14,18]$. However, SoPs of higher-order VV beams and complex polarization patterns produced by coherent superpositions of such vector beams have not been experimentally realized.

In this Letter, we demonstrate the direct visualization of the SoPs of higher-order VV beams and extend it to complex asymmetrical SoPs arising from coherent superpositions of different order vortex beams. We permanently imprint the major axis of complex polarization ellipse distributions on a transparent medium (diamond), through nonlinear light-matter interaction. The resultant self-organized periodic nano-structures (ripples), formed upon irradiation with multiple laser pulses, are oriented parallel to the laser polarization axis, thereby accurately replicating the complex local polarization pattern. We show that the ripple spacing is $\sim 170 \pm 25 \mathrm{~nm}$ and is almost independent of the incident pulse energy, the number of laser pulses, and polarization of light.

In our experiment, different SoPs were generated using beam converters based on birefringent liquid crystal devices called $q$-plates $[19,20]$ with topological charges of $q=$ $+1 / 2$ and -1 . The $q$-plates were tuned by varying the applied voltage to change its optical retardation. At the optimal voltage, the $q$-plates converted linearly polarized Gaussian beams to optical VV beams composed of orbital angular momentum (OAM) states with $\ell= \pm 1$ and $\ell= \pm 2$, respectively [21]. Detuning the individual $q$-plate produced a superposition of partially converted VV beams with the incident Gaussian 
beam. A combination of different $q$-plates resulted in complex asymmetric SoPs due to the superposition of VV beams.

Ultrashort light pulses from a Ti:sapphire laser system (800 nm, $1 \mathrm{kHz}, 45 \mathrm{fs}, 2.5 \mathrm{~mJ} /$ pulse) were focused on a diamond surface $(3 \times 3 \times 0.3 \mathrm{~mm})$ with a 0.25 NA microscope objective. The sample was mounted on three-axis translation stages with a resolution of $100 \mathrm{~nm}$. The incident pulse energies were measured after the microscope objective, thereby taking into account the transmission and reflection losses of all the optics. Pulse energies were varied from $\sim 200-700 \mathrm{~nJ}$ by using a half-wave plate (HWP) and a polarizer. The pulse duration before the microscope objective was $70 \mathrm{fs}$. The laser-ablated regions were characterized by a scanning electron microscope (SEM). The periodicity of the nano-structures was analyzed using 2D fast Fourier transforms.

Figure 1 shows periodic nano-structures produced with radial, azimuthal, and spiral/sink polarizations. These structures were produced by irradiating the diamond surface with 100 laser pulses of energy 280 nJ. Different SoPs were generated by rotating the angle of the incident linear polarization with respect to the $q$-plate (topological charge $=+1 / 2$ ) axis, $\theta$, using a HWP. Radial, azimuthal, and spiral/sink polarizations were obtained for $\theta=0^{\circ}, 90^{\circ}, \pm 45^{\circ}$. SEM images reveal an interesting aspect of the interaction: the emergent ripple pattern is a complete map of the polarization and intensity profile of the VV beams. It accurately reflects the local structure of the electric field, as shown by theoretically simulated polarization patterns in Fig. 1. At low pulse energies, the intensity null at the center of the beam profile results in an unmodified region. However, when using higher pulse energies and a larger number of pulses, the periodic structures extend slightly into the central unablated region.

The periodic structures are oriented parallel to the laser polarization axis and conform with the nano-plasmonic model [22]. The model is based on local field enhancements that arise when the trailing edge of the laser pulse interacts with the plasma generated by the leading edge of the same laser pulse. Inhomogeneous ionization within the focal volume results in randomly formed nano-plasmas that grow into nano-planes with successive laser irradiation. Nano-planes grow along the electric field direction whenever the plasma density is above the critical density. Periodic structures develop over many laser shots, as the growing nano-planes affect the propagation of incident light. From the random distribution, the only planes that are enhanced are the ones that support the propagating modes whose field distributions reinforce their own growth. The periodic structures in the ablated region are spaced by $\sim 170 \pm 25 \mathrm{~nm}$ corresponding to $\lambda / 2 n$, where $n=2.4$ is the refractive index of the medium, and $\lambda$ is the free-space wavelength of the laser beam.

SoPs of different order VV beams and their superposition states were simulated using the Laguerre Gaussian beam:

$$
\begin{aligned}
U(r, \phi, z)= & \frac{C_{\ell p}^{L G}}{w(z)}\left(\frac{r \sqrt{2}}{w(z)}\right)^{|\ell|} \exp \left(\frac{-r^{2}}{w^{2}(z)}\right) L_{p}^{|\ell|}\left(\frac{2 r^{2}}{w^{2}(z)}\right) \\
& \times \exp \left(\frac{-i k r^{2}}{2 R(z)}-i \ell \phi-i k z+i \psi(z)\right),
\end{aligned}
$$

where $p \geq 0$ is the radial index, and $\ell$ is the azimuthal index. $L_{p}^{\ell}$ are the generalized Laguerre polynomials, and $C_{\ell p}^{L G}$ is a normalization constant. $R(z)$ is the radius of curvature of the

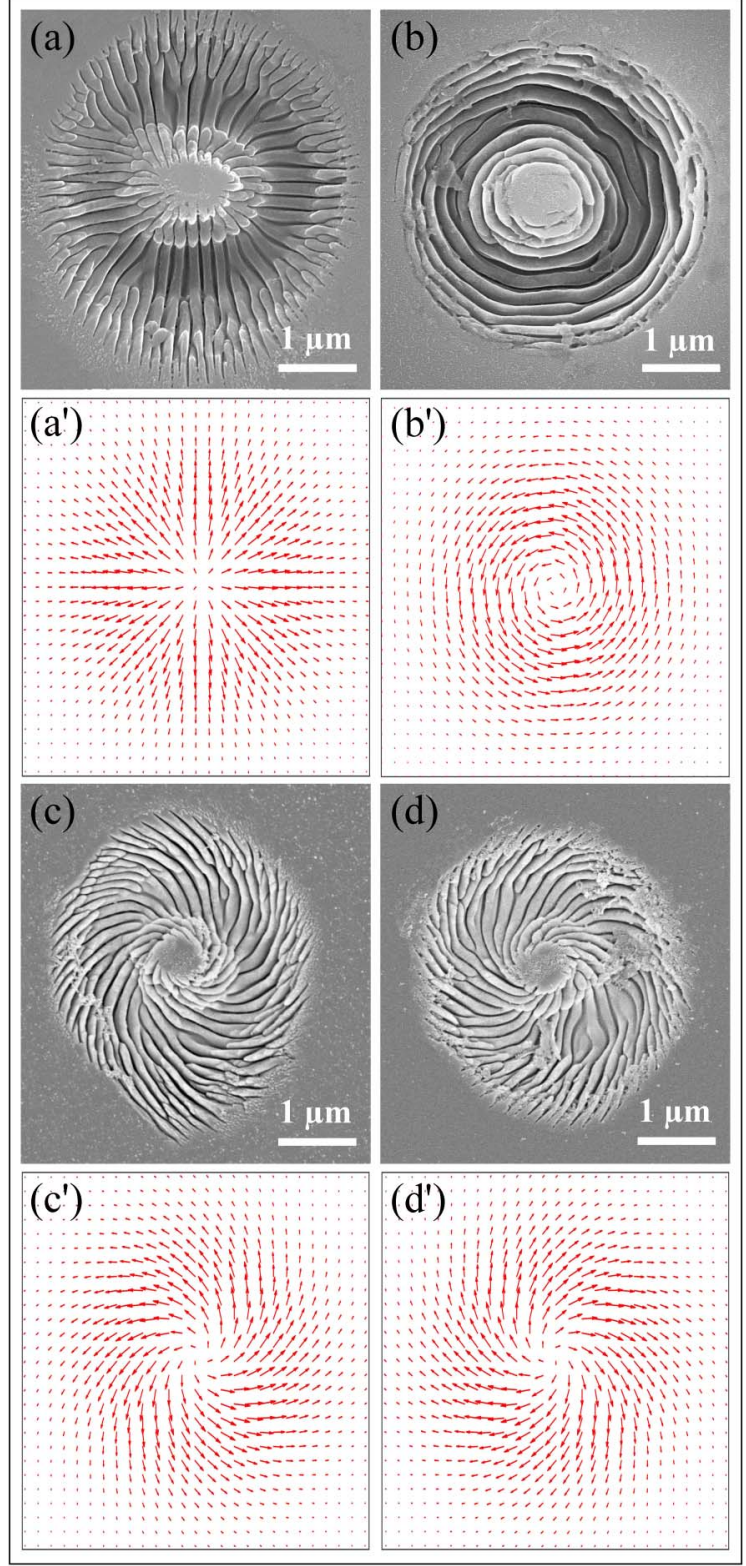

Fig. 1. SEM images showing the mapping of the laser polarization patterns onto the diamond surface in the form of periodic nanostructures. 100 pulses irradiated the sample with an energy of $280 \mathrm{~nJ}$ for (a) radial, (b) azimuthal, (c) counter-clockwise spiral, and (d) clockwise spiral polarization states of a first-order VV beams. $a^{\prime}-d^{\prime}$ are the corresponding expected polarization patterns.

wavefront, $w(z)$ is the beam width, and $\psi(z)$ is the Gouy phase. $r, \phi, z$ are cylindrical coordinates, and $k$ is the wave number.

By decomposing the SoP of the incident light on the $q$-plate into left $\left(\mathbf{e}_{L}\right)$ and right $\left(\mathbf{e}_{R}\right)$ circular polarizations, the polarization state after the $q$-plate is given by

$$
\mathbf{E}_{\text {out }}=\cos \frac{\delta}{2}\left(\begin{array}{c}
\mathbf{e}_{L} \\
\mathbf{e}_{R}
\end{array}\right)+i \sin \frac{\delta}{2}\left(\begin{array}{c}
\mathbf{e}_{R} e^{+2 i\left(q \varphi+\alpha_{0}\right)} \\
\mathbf{e}_{L} e^{-2 i\left(q \varphi+\alpha_{0}\right)}
\end{array}\right),
$$

where $\varphi$ is the azimuthal angle in the beam transverse plane, $\delta$ is the optical retardation, and $\alpha_{0}$ specifies the initial molecular orientation of the liquid crystals with respect to a reference axis. 
A tuned $q$-plate, i.e. $\delta=\pi$, produces a pure OAM carrying beam with $\ell= \pm 2 q$ when exposed to a circularly polarized light. When $\delta \neq \pi$, the $q$-plate produces a coherent superposition of the incident beam with an OAM value of $\ell$ and a converted beam with an OAM value of $\ell= \pm 2 q$ whose relative weights are determined by the parameter $\delta$. The arrows in the simulated SoPs indicate local SoP and the intensity distribution.

Figure 2 shows the mapping of complex polarization patterns formed by VV beams. The first beam [Fig. 2(a)] was produced with a $q$-plate defined by a topological charge of $q=+1 / 2$ followed by a HWP. The resulting polarization pattern is equivalent to the one produced by a $q$-plate with a topological charge of $q=-1 / 2$. The second and third VV beams were produced by a $q$-plate with a topological charge of $q=-1$ [Fig. 2(c)] followed by a HWP [Fig. 2(b)]. Due to an asymmetry in the intensity profile of the beam, there are some regions where the nano-ripples merge to form wider structures.

Periodic nano-structures arising from differently weighted superpositions of Gaussian and VV beams with a topological charge of $q=+1 / 2$ are shown in Fig. 3. Coherent superpositions were obtained by detuning the $q$-plate, such that a partly converted vortex beam co-propagates with the incident linearly polarized Gaussian beam. The superposition of the two beams causes a shift in the null intensity region. The position of the singularity in the intensity profile and, hence, that of the unmodified area within the ablated region shifts from the center with the relative weight between the two beams. As the portion of the Gaussian beam is increased from 30\% to $45 \%$ by correspondingly modifying the voltage applied to the $q$-plate, the position of the singularity shifts more towards the outer region of the ablated area, producing a crescentshaped structure. However, the topological nature of the electric field structure is still preserved.

Complex nano-structures produced by superpositions of $\mathrm{VV}$ beams with a topological charge of $q=-1$ [Fig. 2(c)] and a Gaussian beam are shown in Fig. 4. The singularity (null intensity region) is split and mapped into two distinct regions. This is due to destructive interference in the central vertical region, as the incident linear polarization of the Gaussian beam

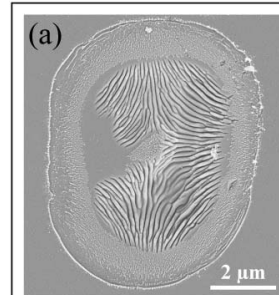

(d)

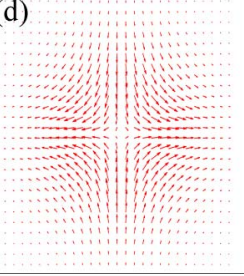

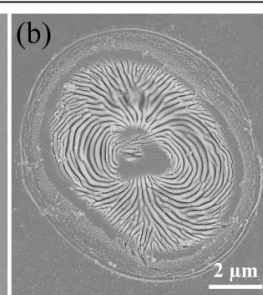

(e)

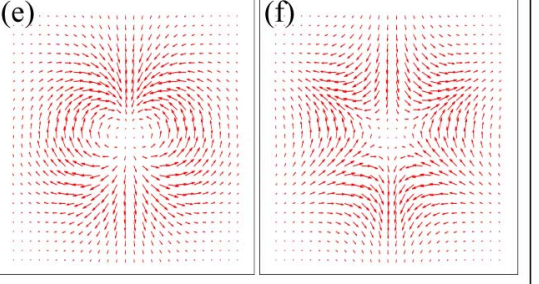

Fig. 2. SEM images of periodic structures formed by $q$-plates with topological charges of (a) $q=-1 / 2$, (b) $q=+1$, and (c) $q=-1$. The corresponding expected polarization patterns are presented in the lower panels (d)-(f). 100 pulses irradiated the sample with a pulse energy of $310 \mathrm{~nJ}$.

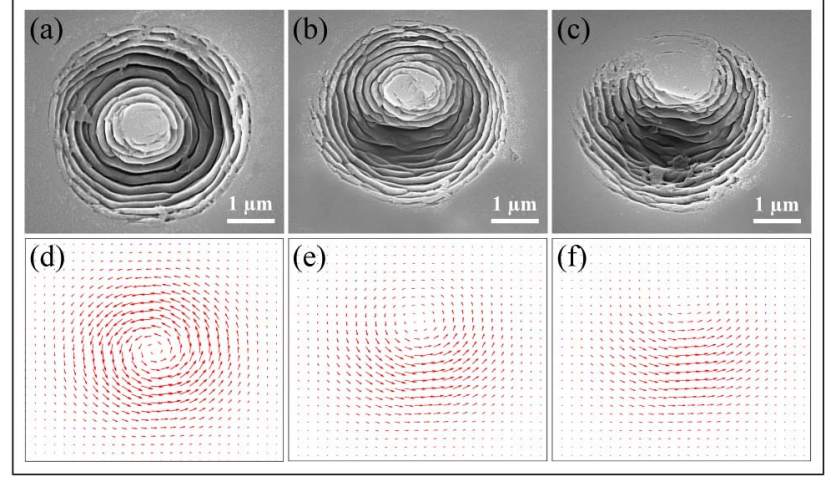

Fig. 3. Superpositions of a linearly polarized Gaussian beam with a VV beam produced by a $q$-plate with a topological charge of $q=+1 / 2$ of differing weights (a) 0:100 (pure VV beam), (b) 30:70, and (c) 45:55. A shift in the position of the singularity with an increasing ratio of the Gaussian component agrees with the expected results in the bottom row. 100 pulses irradiated the sample with a pulse energy of $280 \mathrm{~nJ}$.

is out-of-phase with respect to the VV beam [as shown by arrows in Fig. 2(f)]. The nano-structure orientation is still primarily dictated by the polarization of the VV beam. The spacing between the two null regions increases from 1.21 to $1.95 \mu \mathrm{m}$ by varying the relative weight of the Gaussian beam superimposed on the VV beam.

Figure 5 demonstrates the unique capability of producing distinct nano-structured regions within the ablated area by using superpositions of $\mathrm{VV}$ beams and polarization selection. Figure 5(a) shows periodic nano-structures localized to three regions with distinct orientations. This three petal flower-like structure was obtained by placing a HWP between the $q=$ $-1(\ell= \pm 2)$ and the detuned $q=1 / 2(\ell= \pm 1)$ plates. The HWP reverses the handedness of the polarization ellipse forming the VV beam. After the HWP, the beam incident on the detuned $q=+1 / 2$ plate, defined by $e^{i 2 \varphi} \mathbf{e}_{R}+e^{-i 2 \varphi} \mathbf{e}_{L}$,

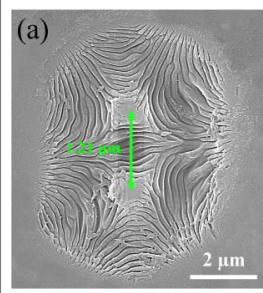

(d)

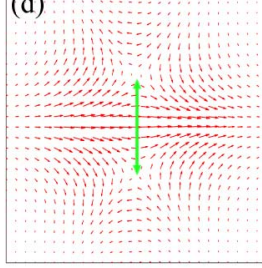

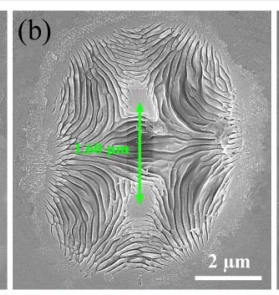

(e)

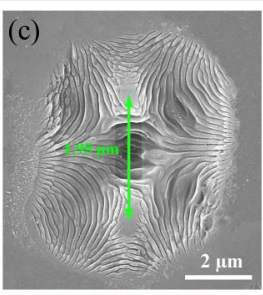

(f)
Fig. 4. Superpositions of linearly polarized Gaussian and VV beams produced by a detuned $q$-plate with a topological charge of $q=-1$ for relative weights of (a), (d) 20:80; (b), (e) 30:70; and (c), (f) 40:60. The top panel shows the SEM images of the nano-structures generated by 50 pulses with an energy of $600 \mathrm{~nJ}$. The bottom panel shows the corresponding polarization patterns. The length of the green arrows indicates the separation between the singularities. 

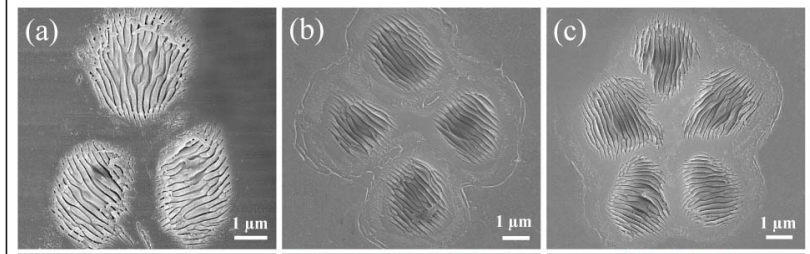

(d)

(e)
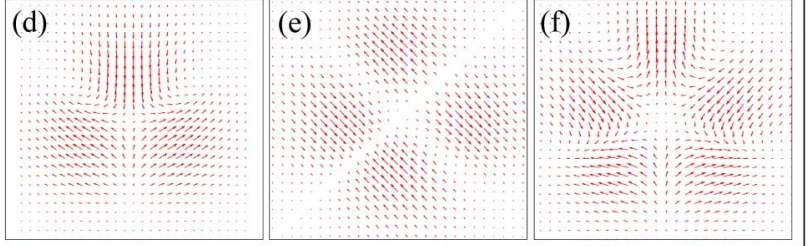

Fig. 5. Mapping of polarization patterns generated by VV beams produced by a combination of (a) $q$-plates with topological charges of $q=-1, q=1 / 2$, and a HWP between (b) a $q$-plate with a topological charge of $q=-1$ and a polarizer (c) $q$-plates with topological charges of $q=-1$ and $q=1 / 2$. All patterns were produced with 50 pulses and an energy of 700 nJ. The bottom panels (e)-(f) show the expected polarization patterns.

undergoes a conversion resulting in the $\alpha\left(e^{i 2 \varphi} \mathbf{e}_{R}+e^{-i 2 \varphi} \mathbf{e}_{L}\right)+$ $\beta\left(e^{i \varphi} \mathbf{e}_{L}+e^{-i \varphi} \mathbf{e}_{R}\right)$ state, which can be interpreted as a coherent superposition of two VV beams.

A four-petal flower-like structure shown in Fig. 5(b) was obtained by placing a polarizer after the $q=-1$ plate. The polarizer introduces diagonal nodes in the intensity profile of the VV beam as in Figs. 2(c) and 2(f) or Figs. 2(b) and 2(e). The orientation of periodic nano-structures in each of the four regions is identical and determined by the axis of the polarizer. Figure 5(c) shows structures obtained by using the same configuration as in Fig. 5(a) without the HWP. The output now corresponds to a superposition state defined by $\alpha\left(e^{i 2 \varphi} \mathbf{e}_{L}+e^{-i 2 \varphi} \mathbf{e}_{R}\right)+\beta\left(e^{i \zeta \varphi} \mathbf{e}_{R}+e^{-i \zeta \varphi} \mathbf{e}_{L}\right)$. The result is a five-petal flower-like structure in which the orientation of nano-structures in each of the five localized regions is different.

Figure 6 shows the periodicity of the nano-ripples as a function of the pulse energy [Fig. 6(a)] and the number of laser pulses [Fig. 6(b)]. Fast Fourier transforms were used to calculate the periodicity of the ripples. While the orientation of the nano-ripples is controlled by the local electric field structure, their spacing seems to have negligible dependence on laser parameters such as pulse energy and the number of pulses. The average spacing is $\sim 170 \pm 25 \mathrm{~nm}$ corresponding to
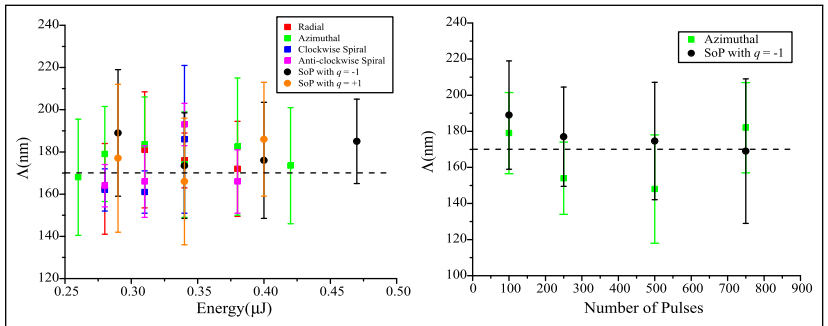

Fig. 6. Periodicity of nano-ripples as a function of (a) pulse energy and (b) number of laser pulses for SoPs corresponding to first- and second-order $\mathrm{VV}$ beams $(q= \pm 1 / 2$ and $q= \pm 1)$, respectively. The dashed line represents the $\lambda / 2 n$ value in both graphs. $\lambda / 2 n$, in accordance with the predictions of the nanoplasmonic model $[1,2]$.

To conclude, we demonstrate the direct visualization of a local electric field structure in the form of periodic nano-ripples by accurately mapping complex polarization patterns of $\mathrm{VV}$ beams onto a sample surface. Superpositions of different order VV beams enabled the controlled fabrication of unconventional, asymmetric surface structures. Configurations discussed in Fig. 5 enabled the fabrication of multiple localized regions of periodic structures in a small area of $<50 \mu \mathrm{m}^{2}$. Further confinement of these regions is possible by focusing the laser beam with a higher numerical aperture objective. In general, the number of such localized regions within the focal area of the laser increases with the order of the VV beams being superimposed. Each localized region will exhibit different degrees of birefringence owing to different orientations of the periodic nano-structures within the region. Therefore, our findings have potential technological significance.

Funding. Natural Sciences and Engineering Research Council of Canada (NSERC); Canada Research Chairs; Canadian Foundation for Innovation; Ontario Ministry of Economic Development and Innovation.

\section{REFERENCES}

1. V. R. Bhardwaj, E. Simova, P. P. Rajeev, C. Hnatovsky, R. S. Taylor, D. M. Rayner, and P. B. Corkum, Phys. Rev. Lett. 96, 057404 (2006).

2. Y. Shimotsuma, P. G. Kazansky, J. Quu, and K. Hirao, Phys. Rev. Lett. 91, 247405 (2003).

3. P. B. Corkum and F. Krausz, Nat. Phys. 3, 381 (2007).

4. G. Vampa, T. J. Hammond, N. Thiré, B. E. Schmidt, F. Légaré, C. R. McDonald, T. Brabec, and P. B. Corkum, Nat. Phys. 522, 462 (2015).

5. K. Nakanishi, N. Berova, and R. Wood, Circular Dichroism: Principles and Applications (John Wiley \& Sons, 1994).

6. C. Lux, M. Wollenhaupt, T. Bolze, Q. Liang, J. Köhler, C. Sarpe, and T. Baumert, Angew. Chem. (Int. Ed.) 51, 5001 (2012).

7. L. Allen, M. W. Beijersbergen, R. J. C. Spreeuw, and J. P. Woerdman, Phys. Rev. A 45, 8185 (1992).

8. V. Marceau, C. Varin, and M. Piché, Opt. Lett. 38, 821 (2013).

9. R. Dorn, S. Quabis, and G. Leuchs, Phys. Rev. Lett. 91, 233901 (2003). 10. B. J. Roxworthy and K. C. T. Jr., New J. Phys. 12, 073012 (2010).

11. H. Poincaré, Théorie mathématique de la lumière (Gauthiers-Villars, 1892), Vol. 2, p. 318.

12. F. Cardano, E. Karimi, S. Slussarenko, L. Marrucci, C. de Lisio, and E. Santamato, Appl. Opt. 51, C1 (2012).

13. C. Hnatovsky, V. Shvedov, W. Krolikowski, and A. Rode, Phys. Rev. Lett. 106, 123901 (2011).

14. J. J. Nivas, F. Cardano, Z. Song, A. Rubano, R. Fittipaldi, A. Vecchione, D. Paparo, L. M. R. Bruzzese, and S. Amoruso, Sci. Rep. 7, 42142 (2017).

15. J. Bonse, J. Krüger, S. Höhm, and A. Rosenfeld, J. Laser Appl. 24 042006 (2012).

16. Y. Jin, O. J. Allegre, W. Perrie, K. Abrams, J. Ouyang, E. Fearon, S. P. Edwardson, and G. Dearden, Opt. Express 21, 25333 (2013).

17. K. K. Anoop, A. Rubano, R. Fittipaldi, X. Wang, D. Paparo, A. Vecchione, L. Marrucci, R. Bruzzese, and S. Amoruso, Appl. Phys. Lett. 104, 241604 (2014).

18. E. M. Hsu, N. A. Mailman, G. A. Botton, and H. K. Haugen, Appl. Phys. A 103, 185 (2011).

19. L. Marrucci, C. Manzo, and D. Paparo, Phys. Rev. Lett. 96, 163905 (2006).

20. H. Larocque, J. G. Bischoff, F. Bouchard, R. Fickler, J. Upham, R. Boyd, and E. Karimi, J. Opt. 18, 124002 (2016).

21. L. Marrucci, E. Karimi, S. Slussarenko, B. Piccirillo, E. Santamato, E. Nagali, and F. Sciarrino, J. Opt. 13, 064001 (2011).

22. V. R. Bhardwaj, P. P. Rajeev, P. B. Corkum, and D. M. Rayner, J. Phys. B 39, S397 (2006). 